\title{
Cancro resinoso del pino: ¿una amenaza potencial para las plantaciones de Pinus radiata en Chile?
}

\section{Pitch canker, a potential threat to Pinus radiata plantations in Chile?}

\author{
Angela Carrasco $^{1 *}$, Eugenio Sanfuentes ${ }^{1}$, Álvaro Durán $^{2,3}$ \& Sofía Valenzuela ${ }^{1 *}$ \\ ${ }^{1}$ Universidad de Concepción, Centro de Biotecnología y Facultad de Ciencias Forestales, Victoria 631, Concepción, Chile. \\ ${ }^{2}$ Bioforest S.A., Camino a Coronel km 15, Concepción, Chile. \\ ${ }^{3}$ Ontario Forest Research Institute, 1235 Queen St. E, Sault Ste Marie, ON P6A 2E5, Canadá. \\ *angelacarrasco@udec.cl; sofvalen@udec.cl
}

\begin{abstract}
RESUMEN
Fusarium circinatum es el hongo causante de la enfermedad "cancro resinoso" (pitch canker), que afecta numerosas especies de coníferas. En esta revisión se describe el estado del arte de F. circinatum a nivel mundial y su situación actual en Chile, abarcando antecedentes del patógeno y de la enfermedad. En Chile, el patógeno fue detectado en setos de Pinus radiata a fines de 2001, encontrándose hoy presente en 39 viveros de un total de 196, ubicados entre las regiones de O'Higgins y de Los Ríos. Después de 14 años de la detección de F. circinatum en Chile, el patógeno no ha presentado diseminación en las plantaciones comerciales de $P$. radiata. Se destacan las medidas para el manejo integrado de la enfermedad, donde la resistencia genética sería la principal estrategia ante un escenario en que el cancro resinoso se presente en las plantaciones comerciales de $P$. radiata en Chile.
\end{abstract}

Palabras clave: Fusarium circinatum, enfermedades en viveros, resistencia genética.

\begin{abstract}
Fusarium circinatum is the fungus that causes the disease "canker" (pitch canker), which affects many species of conifers. This review describes the state of the art of F. circinatum worldwide and the current situation in Chile, covering the history of the pathogen and the disease. In Chile, the pathogen was detected in Pinus radiata hedges in late 2001, being present today in 39 of 196 nurseries, located between the regions of O'Higgins and Los Ríos. After 14 years of the detection of $F$. circinatum, the pathogen has not attacked commercial plantations of $P$. radiata. Measures for integrated disease management, where genetic resistance constitutes the main strategy in a scenario in which pitch canker could appear in commercial plantations of $P$. radiata in Chile.
\end{abstract}

KeYwoRDs: Fusarium circinatum, nursery diseases, genetic resistant.

\section{INTRODUCCIÓN}

El cancro resinoso del pino (pitch canker) es causado por el hongo ascomicete Fusarium circinatum Nirenberg et O'Donell (telomorfo $=$ Gibberella circinata Nirenberg et O'Donell) (Nirenberg \& O'Donnell 1998). El nombre de la enfermedad se debe a la abundante resinación que produce el patógeno en el árbol infectado. El patógeno ha sido detectado en USA (Hepting \& Roth, 1946), Haití (Hepting \& Roth 1953), Japón (Kobayashi \& Muramoto 1989), Sudáfrica (Viljoen et al. 1994), México (Guerra-Santos 1999), España (Landeras et al. 2005), Italia (Carlucci et al. 2007), Francia (EPPO 2006), Portugal (Bragança et al. 2009), Uruguay (Alonso \& Bettucci 2009), Corea del
Sur (Woo et al. 2010), Colombia (Steenkamp et al. 2012) y Brasil (Pfenning et al. 2014), afectando a más de 60 especies del género Pinus y Pseudotsuga menziesii Mirb. Franco (Hodge \& Dvorak 2000, Wingfield et al. 2008). Los primeros reportes indicaron que la enfermedad afectaba árboles de mediana edad y adultos, sin embargo, después se determinó que puede ocurrir en cualquier etapa del ciclo de vida del hospedero (Dwinell et al. 2001).

Esta enfermedad causa pérdidas en plantaciones comerciales, viveros $\mathrm{y}$ huertos semilleros, siendo ocasionalmente un problema en bosques nativos de especies de Pinus. A nivel mundial, el cancro resinoso es considerado como una amenaza a las plantaciones de Pinus spp., debido a que la mayoría de las especies de interés comercial son 
susceptibles a $F$. circinatum (Wingfield et al. 2008). Entre las especies más susceptibles se encuentra Pinus radiata D. Don, siendo esta especie una de las coníferas más plantadas del hemisferio sur (Gordon et al. 2001, Wingfield et al. 2008). Las pérdidas causadas por esta enfermedad se deben principalmente a una reducción en la calidad de la madera por deformaciones de las ramas y troncos, así como también a la mortalidad de árboles y plántulas de viveros (Storer $e t$ al. 2002).

En Chile, F. circinatum se identificó por primera vez en el año 2001, causando la muerte de setos en contenedores y raíz desnuda de $P$. radiata, en viveros de la Región del Biobío (Wingfield et al. 2002). Posteriormente, el patógeno continuó siendo detectado en plantas y setos en viveros entre las regiones del Libertador General Bernardo O’Higgins y de Los Ríos. Además, F. circinatum ha sido diagnosticado en árboles aislados en plantaciones comerciales jóvenes de $P$. radiata (1 a 4 años de edad), en las regiones del Maule y del Biobío, probablemente como consecuencia de la utilización de plantas asintomáticas e infectadas por el patógeno en vivero (Beéche et al. 2005). Prospecciones sanitarias efectuadas por las empresas forestales, así como estudios realizados en la última década, no han demostrado una diseminación posterior del patógeno en estas plantaciones, siendo hasta ahora un problema circunscrito exclusivamente a los viveros (Wingfield et al. 2002, Jacobs et al. 2007, Proyecto Fondo SAG 2007)

La diseminación de $F$. circinatum preocupa a países libres de la enfermedad, como Nueva Zelanda y Australia, constituyéndose en una seria amenaza económica para la industria forestal, pronosticándose pérdidas de hasta $20 \%$ en la producción de la madera (Cook \& Matheson 2008). En Australia, un estudio estableció que mantener los bosques de $P$. radiata libres de $F$. circinatum en los próximos 30 años, producirían un beneficio de al menos 3 millones de dólares anuales, lo que es equivalente a pérdidas del $0,4 \%$ de la producción anual de $P$. radiata en este país (Cook \& Matheson 2008), evidenciándose el impacto potencial que este patógeno tendría al ingresar a una nueva área geográfica.

Los antecedentes de los últimos años en Chile indicarían que presumiblemente la patología aún está en etapas tempranas de su establecimiento, no constituyéndose aún este patógeno en un problema importante para las plantaciones forestales en el país. Hasta el momento el comportamiento de la enfermedad es similar a lo descrito en Sudáfrica, donde el patógeno fue detectado en viveros en 1990 (Viljoen et al. 1994), y después de 15 años la enfermedad comenzó a extenderse en plantaciones de Pinus patula Schltdl. \& Cham., ocasionando pérdidas importantes para la industria forestal (Coutinho et al. 2007).

Esta revisión resume y discute la situación actual de F. circinatum en Chile y revisa el conocimiento generado a nivel mundial, para justificar que este patógeno podría ser una amenaza potencial para las plantaciones de $P$. radiata en Chile. Se propone, al igual que en otros países, que para reducir el impacto del cancro resinoso en plantaciones comerciales de $P$. radiata en nuestro país, se debe implementar una estrategia de manejo integrado de la enfermedad, la cual debe incluir medidas cuarentenarias, control cultural, químico y biológico, y resistencia genética.

SíNTOMAS Y SIGNOS DE LA ENFERMEDAD

Fusarium circinatum puede infectar hospederos susceptibles en cualquier etapa de su ciclo de vida y a diferentes órganos de la planta, tales como: brotes, tallos, ramas, acículas, conos, semillas y raíces expuestas al patógeno (Dwinell et al. 2001, Wingfield et al. 2008). La expresión de los síntomas dependerá del hospedero, órgano afectado y las condiciones ambientales (Dwinell et al. 1985, Gordon et al. 2001).

Cuando el patógeno infecta plántulas, el diagnóstico puede ser difícil, ya que los síntomas son similares a los causados por otras enfermedades ocasionadas por otros hongos (Viljoen et al. 1994, Gordon et al. 2001), siendo necesario realizar un diagnóstico en laboratorio utilizando técnicas de PCR (Polymerase Chain Reaction) (Schweigkofler et al. 2004, Britz et al. 2005). La enfermedad se manifiesta con acículas cloróticas o de color marrónrojizo y marchitamiento (Fig. 1a) (Dwinell et al. 2001), además causa damping-off de pre y post emergencia (Viljoen et al. 1994). En damping-off de pre-emergencia, la cubierta de las semillas y los cotiledones son colonizados por el hongo. En el caso del damping-off de post-emergencia (Fig. $1 b$ ), ocurre necrosis en el cuello de la plántula, resultando en clorosis y muerte (Viljoen et al. 1994). En plantas en vivero (mayores de seis meses) el patógeno se puede asociar a pudrición de raíces (Gordon et al. 2001), los principales síntomas causados por la infección de $F$. circinatum incluyen marchitamiento apical (Dwinell et al. 2001), seguido por una decoloración púrpura en el punto de infección. A medida que el patógeno continúa colonizando, el follaje de las plantas adquiere un color marrón y posteriormente muere. En estado avanzado de la enfermedad pueden presentarse esporodoquios en el tallo de las plantas (Wingfield et al. 2008) (Fig. 1c). Este patrón sintomatológico es diferente al del cancro resinoso en árboles adultos, caracterizado por un flujo masivo de resina, denominándose al problema en vivero como "enfermedad de F. circinatum" (Mitchell et al. 2011) o "muerte por Fusarium". Una situación particular se presenta en las plantas madres o setos, las cuales poseen mayor susceptibilidad al patógeno (Fig. 1d, 1e y 1f), condición que sería consecuencia de un constante estrés de la planta, por la permanente colecta de estacas (Mitchell et al. 2011). En los setos, la enfermedad se manifiesta con marchitez de los brotes y decoloración del follaje producto de la infección causada por el patógeno en el cuello de la planta (Wingfield et al. 2002, Mitchell et al. 2011).

En árboles adultos, los síntomas en ramas comienzan usualmente con marchitamiento y decoloración de las 
acículas, tornándose luego de color rojizo, seguido de defoliación y muerte progresiva (Storer et al. 2002). La muerte del tejido (cancro) en la zona infectada causa un anillamiento y la consecuente obstrucción del flujo de agua, causando finalmente la muerte de la rama. A su vez, en la zona de la infección se produce resina y la madera toma un color miel, que se observa al retirar la corteza sobre la lesión (Gordon et al. 2001, Storer et al. 2002).
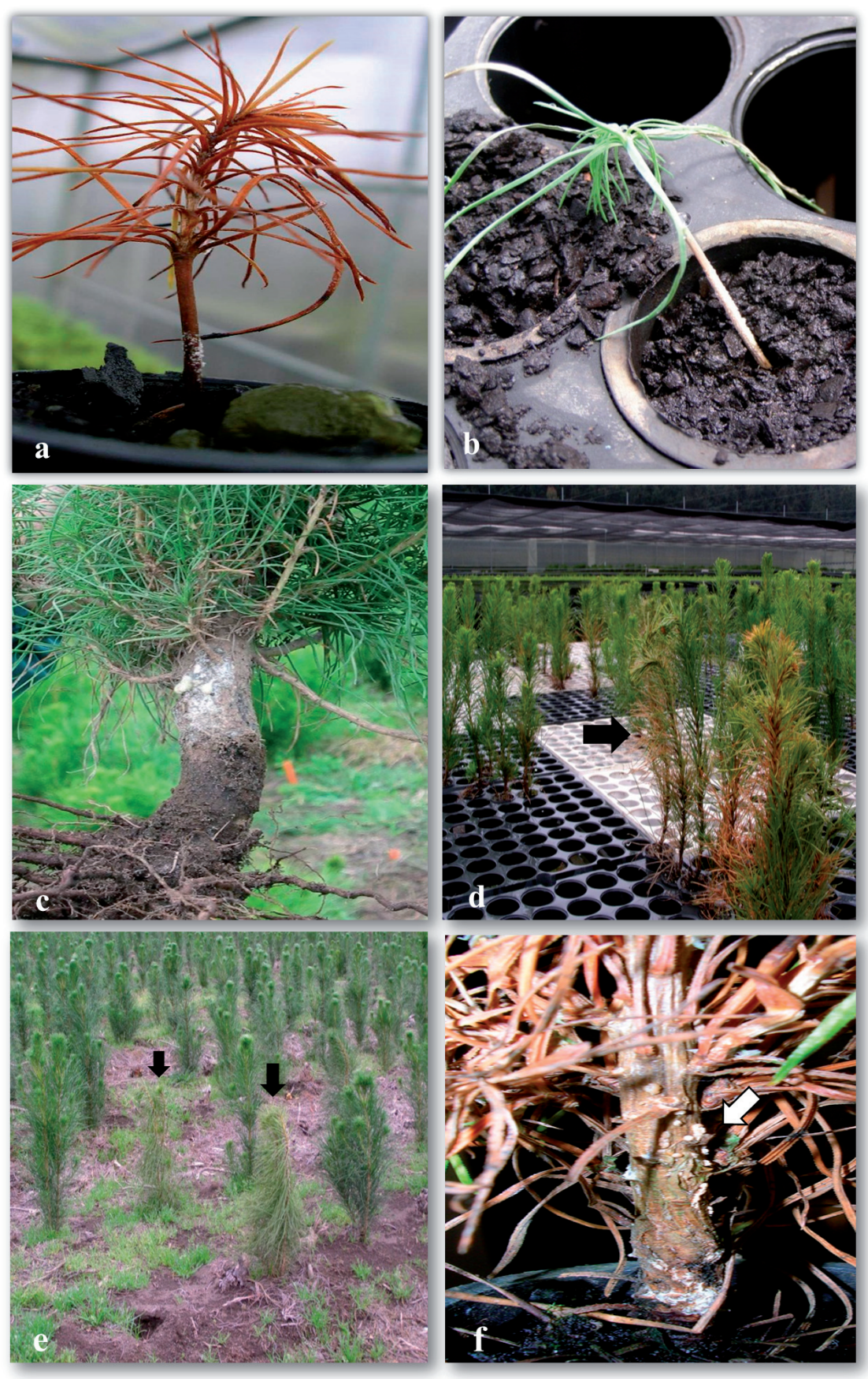

Figura 1. Síntomas y signos de Fusarium circinatum en viveros de Pinus radiata. a: cancro en tallo de plántula; b: damping-off en plántulas; c: cancro y resinación en cuello de planta de seto; d: marchitamiento apical en plantas a raíz cubierta; e: marchitamiento de follaje en plantas de setos; f: esporodoquios en tallo (Fotos a y f proporcionadas por G. González, fotos b-e proporcionadas por E. Sanfuentes).

Figure 1: Symptoms and signs of Fusarium circinatum in Pinus radiata seedlings and plants in nursery. a: stem lesion and wilting on seedling; b: damping-off in seedling c: canker and resin in collar; d: apical wilting; e: foliage wilting and discoloration; f: sporodochia in stem (Picture a and f provided by G. González, picture b-e provided by E. Sanfuentes). 
El patógeno también puede infectar órganos reproductivos, causando la muerte de conos femeninos y maduros, deteriorando las semillas (Dwinell et al. 2001). Los conos en las ramas infectadas pueden abortar, si la infección ocurre antes de completar su desarrollo, no obstante, la infección en ramas también puede suceder después que los conos han madurado. Los conos de Pinus taeda $\mathrm{L}$. infectados por el patógeno se deforman y presentan menor tamaño, algunos presentan necrosis en la zona apical y una resinación interna. La infección interna de conos no necesariamente se correlaciona con los síntomas externos, de esta forma conos aparentemente sanos pueden también estar infectados (Storer et al. 1998, Dwinell et al. 2001). El patógeno también puede infectar semillas interna o externamente, causando el deterioro de la semilla, aunque con frecuencia los síntomas se presentan solo al momento de la germinación (Storer et al. 1998).

\section{TAXonomía de FusARIUM CIRCINATUM}

Fusarium circinatum fue detectado por primera vez en Pinus virginiana Mill. en el año 1946, siendo identificado como una especie no descrita del género Fusarium, ubicada en la sección Liseola (Hepting \& Roth 1946). Luego, en 1949 el hongo fue descrito como Fusarium lateritium Nees f. sp. pini Hepting en la sección Lateritium (Snyder et al. 1949) y en 1978, como F. moniliforme J. Sheld. var. subglutinans Wollenweb. \& Reinking en la sección Liseola (Kuhlman 1982). En 1983, fue elevado a nivel de especie denominándose Fusarium subglutinans (Wollenweb \& Reinking) Nelson, Toussoun \& Marasas (Nelson et al. 1983) y en 1992 se clasificó como $F$. subglutinans f. sp. pini Correll, Gordon, McCain, Fox, Koehler, Wood \& Schultz (Correll et al. 1992, Huss et al.1996). Nirenberg \& O'Donnell, en 1998, lo describieron con el nombre de F. circinatum Nirenberg \& O'Donnell, perteneciente al complejo Gibberella fujikuroi (Sawada) Wollenw., dentro de la sección Liseola, confirmado por estudios morfológicos (Britz et al. 2005) y análisis filogenéticos (Schweigkofler et al. 2004), siendo designado a la población de apareamiento H (Britz et al. 2005).

\section{Ciclo Biológico y Epidemiología del Patógeno}

Existe poco conocimiento con respecto a la biología y ecología del hongo, pero se han conducido estudios sobre la dinámica de inóculo, dispersión, infección, colonización, sobrevivencia y reproducción sexual del patógeno (Wingfield et al. 2008). El inóculo de $F$. circinatum puede estar disponible en todas las estaciones del año (Wingfield et al. 2008). Los conidios se dispersan por medio del viento, insectos vectores, salpicadura de agua, suelo y por el movimiento de plantas infectadas a nuevas áreas (Storer et al. 1998, Gordon et al. 2001, Wingfield et al. 2008). El patógeno infecta tejido leñoso del árbol a través de heridas provocadas por insectos, el hombre o heridas naturales.
En tallos de Pinus densinflora Sieb. \& Zucc. y P. rigida Mill., la mayoría de las microconidias germinan $12 \mathrm{~h}$ post inoculación, formando tubos germinativos que penetran al tejido del huésped a través de aberturas naturales del tallo (Thoungchaleun et al. 2008). No existe evidencias de que el hongo se pueda movilizar en forma sistémica en la planta, significando de esta manera que cada cancro o lesión es producto de una infección independiente (Gordon et al. 2001).

Las conidias están presentes en restos vegetales del árbol, y sus cuerpos de fructificación perduran durante meses en brotes enfermos, produciendo grandes cantidades de conidias, desde donde se pueden iniciar nuevas infecciones. A pesar de que $F$. circinatum no produce clamidosporas, es capaz de sobrevivir durante varios meses en suelo húmedo y al menos un año en suelo seco, y hasta por tres años en suelo almacenado bajo refrigeración (Wingfield et al. 2008). Debido a esta capacidad de permanecer en el suelo puede actuar como un patógeno de raíces (Dwinell et al. 1985). En plantaciones, el movimiento de material infectado es la causa más probable de la dispersión del agente. En vivero este hongo se disemina por el aire y también por la introducción de semillas infectadas por el hongo, por herramientas contaminadas y por el hombre (Dwinell et al. 1985, Wingfield et al. 2008).

Factores de estrés como la sequía, exceso de fertilización, temperaturas elevadas y anegamiento favorecen una mayor incidencia de la enfermedad (Wingfield et al. 2008, Gordon et al. 2015). Es así como la mayor severidad del cancro resinoso ocurre en el SE de EE.UU., donde las precipitaciones son comunes durante los períodos cálidos. Por el contrario, en California, donde la mayoría de las zonas reciben precipitaciones sólo durante los meses más fríos del año, el cancro resinoso se limita a la costa, donde las temperaturas moderadas coinciden con alta humedad y/o condensación proporcionada por el húmedo aire marino (Gordon et al. 2015).

\section{DiversidAD GENÉTICA DE FUSARIUM CIRCINATUM}

Fusarium circinatum es un hongo heterotálico que puede reproducirse sexual y asexualmente (Britz et al. 2005). El estado sexual del hongo (Gibberella circinata Nirenberg \& O’Donnell ex Britz, T.A. Cout., M.J. Wingf. \& Marasas.) no ha sido observado en la naturaleza, pero se ha producido en condiciones de laboratorio (Correll et al. 1992, Wikler \& Gordon 2000). En los primeros estudios de diversidad genética de $F$. circinatum, se determinaron cinco grupos de compatibilidad vegetativa (GCVs) en California y 45 en Florida (Correll et al. 1992). Esta limitada diversidad genética de $F$. circinatum en California y la ausencia de reproducción sexual es consistente con una reciente introducción del patógeno. Por otro lado, la alta diversidad de los aislamientos en Florida implicaría una historia de autocruzamiento entre las poblaciones del patógeno, 
además de corresponder a GCVs diferentes (Correll et al. 1992). Sin embargo, con el uso de marcadores moleculares se evidenció la existencia de dos haplotipos multilocus que eran compartidos entre ambas poblaciones, proponiendo que nuevos GCVs pueden surgir por mecanismos distintos a la recombinación sexual (Correll et al. 1992, Wikler \& Gordon 2000). En Sudáfrica fueron determinados 23 GCVs (para 69 aislamientos) y presentando los dos tipos de apareamiento (MAT-1 y MAT-2) (Britz et al. 2005). Este número de GCVs no sería concordante con una reciente introducción de la especie, sugiriendo que la variabilidad del patógeno en este país es producto de múltiples introducciones de diversos genotipos además de reproducción sexual, resultando en la segregación de múltiples loci incompatibles vegetativamente (Britz et al. 2005).

El análisis de ocho loci polimórficos de aislamientos provenientes de California y Sureste de EE.UU., Sudáfrica, México y Japón, establecieron que existe una mayor diversidad genética en México, coincidiendo con la hipótesis de ser el centro de origen de $F$. circinatum, como también el centro de diversidad de Pinus spp. (Perry et al. 1998, Wikler \& Gordon 2000). Los aislamientos de F. circinatum de California y sureste de EE.UU. y Japón presentaban una pequeña distancia genética correspondiendo a un mismo genotipo. Estos resultados indicarían que el patógeno fue dispersado probablemente desde el sureste de EE.UU. a California y luego hacia Japón, o directamente desde el Sureste de EE.UU. Por otra parte, existiría una mayor conexión entre las poblaciones de $F$. circinatum de México y Sudáfrica (Wikler \& Gordon 2000), sugiriendo que el patógeno fue introducido a Sudáfrica a través de semillas contaminadas desde México (Berbegal et al. 2013). En el norte de España las poblaciones de $F$. circinatum están estructuradas en dos grupos divergentes y presentan un baja diversidad genética, concordando con la hipótesis que el patógeno fue introducido en al menos dos oportunidades, probablemente desde EE.UU. (Iturritxa et al. 2011, Berbegal et al. 2013).

Control de la enfermedad: A pesar de la importancia de la enfermedad a nivel mundial, no existen medidas de control individuales completamente eficaces contra $F$. circinatum en viveros y plantaciones forestales. Para reducir el impacto de la enfermedad se debe considerar el manejo integrado, incluyendo medidas cuarentenarias, control cultural, químico y biológico, y selección de árboles con resistencia genética (Dwinell et al. 1985, 2001, Wingfield et al. 2008).

CONTROL CULTURAL: La principal estrategia de manejo se basa en la reducción de las fuentes de inóculo, a través de la eliminación de material infectado (Dwinell et al. 1985). Para reducir la población del patógeno en vivero, es necesario incrementar los niveles de higiene, tales como: utilizar agua de riego libre de patógeno, bandejas y contenedores desinfestados y eliminar y quemar las plántulas con síntomas del patógeno (Dwinell et al. 1985, Wingfield et al. 2008).

En regiones donde las plantaciones presentan altos niveles de la enfermedad, es recomendable excluir la utilización de especies susceptibles al patógeno. La reducción del inóculo, incluye la remoción y eliminación de los árboles infectados y restricción del movimiento de madera infectada. La poda de ramas infectadas no ha sido eficaz como medida preventiva para confinar al patógeno en el árbol, sin embargo, en árboles con menor infección y distantes de árboles enfermos, esta operación podría disminuir el desarrollo de un foco de enfermedad. La desinfestación de las herramientas de poda, con cloruro de benzalconio, hipoclorito de sodio o de calcio al $1 \%$ o peróxido de hidrógeno al $10 \%$, antes y después de las operaciones de poda, pueden reducir la dispersión de la enfermedad (Storer et al. 2002).

Otra estrategia para prevenir la presencia o la propagación del cancro resinoso del pino en plantaciones, es evitar las podas en épocas de máxima esporulación. Prácticas culturales como fertilización, riego, densidad de la plantación, y control de malezas, influyen en la incidencia y severidad de la enfermedad (Dwinell et al. 1985). En plantaciones de Pinus elliottii Engelm. var. elliottii fertilizadas con nitrógeno, los árboles han presentado cancros de mayor longitud y una mayor tasa de progreso de la enfermedad (Fraedrich \& Witcher 1982).

Control Químico y BIOLÓGico: Actualmente, existen pocos productos químicos registrados que permitan el control eficaz de $F$. circinatum, y algunos de éstos tienen restricciones de uso por normas de certificación ambiental (FSC 2007). Entre los fungicidas empleados para el control del patógeno están: procloraz, tebuconazole, benomyl, captan, difenoconazole y propiconazole (Dwinell et al. 1985, Wingfield et al. 2008, Mitchell et al. 2011). En estudios in vitro los fungicidas más eficaces en la inhibición de $F$. circinatum han sido procloraz y tebuconazol (TPCP 2002). El empleo de insecticidas podrían reducir los niveles del cancro resinoso, al minimizar la propagación de la enfermedad a través de insectos (Dwinell et al. 1985). Es así como en plantaciones de $P$. taeda L. la aplicación de carbofurano redujo la incidencia de daños por insectos y también el número de árboles infectados por $F$. circinatum (Runion et al. 1993).

La recolección de semillas no es recomendable en las zonas donde $F$. circinatum esté presente. Una alternativa es desinfectar las semillas provenientes de huertos semilleros durante 15 min con peróxido de hidrogeno al $30 \%$, a fin de minimizar potenciales infecciones. Este compuesto puede penetrar la cubierta de la semilla, produciendo escarificación, aumentando la tasa de germinación (Wingfield et al. 2008).

El uso de microorganismos, individualmente o en 
combinación con otras medidas de control, ha permitido reducir el uso de pesticidas en otros patosistemas, convirtiéndose en una estrategia de control con gran potencial (Moraga-Suazo et al. 2011). Los estudios sobre el control biológico de F. circinatum aún son escasos y se basan principalmente en la selección de antagonistas in vitro. Algunas excepciones son los estudios de BarrowsBroaddus \& Kerl (1981) y Moraga-Suazo et al. (2011). En el primero, en un ensayo en condiciones de invernadero, aislados de Arthrobacter spp. redujeron la esporulación del patógeno en el sitio de infección y en el segundo, aislados de Clonostachys rosea (Link:Fr.) Schroers, Samuels, Seifert \& Gams y Trichoderma spp, redujeron la incidencia de damping-off por F. circinatum en plántulas de $P$. radiata. Recientemente Martínez-Álvarez et al. (2016) describieron que Chaetomium aureum (Chivers) y Alternaria sp. disminuyeron la severidad del daño causado por $F$. circinatum en plántulas de $P$. radiata, concluyendo que estas especies endófitas pueden ser potenciales agentes de control biológico.

Resistencia genética: La mayoría de los estudios de resistencia genética en $P$. radiata se basan en observaciones de campo y ensayos de inoculación en condiciones controladas en invernadero (Hodge \& Dvorak 2000, Storer et al. 2002). Se ha determinado que las especies de pino varían ampliamente en el nivel de susceptibilidad a $F$. circinatum, siendo $P$. radiata, Pinus greggii Engelm. y $P$. patula clasificados como susceptibles y Pinus tecunumanii Schw. Perry, Pinus oocarpa Schiede y Pinus jaliscana Perez de la Rosa, entre los más resistentes (Hodge \& Dvorak 2000). La mayoría de las especies de pinos nativos de California parecen ser susceptibles al cancro resinoso, aunque la severidad puede variar ampliamente entre los individuos (Gordon et al. 2001), como en el caso de P. radiata, aunque esta variabilidad contrasta con la alta susceptibilidad que han presentado los genotipos en plantaciones comerciales de esta especie (Storer et al. 1998). Esta variación en susceptibilidad también se ha constatado entre genotipos de $P$. taeda en huertos semilleros, donde algunos genotipos después de inoculados con $F$. circinatum no han manifestado la enfermedad (Kayihan et al. 2005, Roux et al. 2007). Estos casos indican la existencia de variación genética en los hospederos, la que puede ser utilizada en programas de mejoramiento genético, para seleccionar genotipos resistentes como una medida de control (Dwinell et al. 1985, Roux et al. 2007).

La resistencia genética a $F$. circinatum se ha demostrado en más de 23 especies de pinos (Viljoen et al. 1994, Hodge \& Dvorak 2000, Roux et al. 2007), aunque los mecanismos de resistencia aún son desconocidos. En especies de Pinus $s e$ ha sugerido que la resistencia tendría una naturaleza poligénica (Kayihan et al. 2005, Matheson et al. 2006, Quesada et al. 2010), en la que participarían genes que codifican para proteínas relacionadas con la patogenicidad o PR (quitinasas, peroxidasas clase III, ribonucleasas, taumatina), fenilalanina amonio liasa (PAL), pinosilvin sintasa (PST), fenilcumarín bencílico éter reductasa (PCBER) y proteínas antimicrobianas (Morse et al. 2004, Fitza et al. 2013, Donoso et al. 2015). Recientemente, la búsqueda de QTLs (Quantitative Trait Locus) (MoragaSuazo et al. 2014) y mapeo asociativo (Quesada et al. 2010) han permitido encontrar loci de carácter cuantitativo asociados a la resistencia contra $F$. circinatum.

La resistencia genética en el tiempo está estrechamente relacionada con la virulencia del patógeno y la factibilidad que las poblaciones de F. circinatum generen nuevos patotipos (Gordon et al. 2006). En poblaciones de F. circinatum se ha determinado que una variación en la virulencia del patógeno como consecuencia de la reproducción sexual podría incrementar la posibilidad de generar nuevos genotipos del patógeno capaces de superar los niveles de resistencia presentes en las poblaciones hospederas (Gordon et al. 2006, Wingfield et al. 2008, Gordon et al. 2015). Esta situación se ve respaldada por el reciente estudio de Slinski et al. (2016), donde determinaron que la heredabilidad de la virulencia en patotipos de $F$. circinatum de California es alta $\left(h^{2}=0,74\right)$, sugiriendo que tiene un fuerte control genético.

Para incrementar los niveles de resistencia en pinos a $F$. circinatum, se pueden emplear varias estrategias de selección y cruzamientos a largo plazo, dado que la resistencia al patógeno en genotipos específicos se encuentra bajo fuerte control genético (Gordon et al. 2001, Storer et al. 2002). Se ha constatado una alta heredabilidad de esta resistencia en familias de $P$. radiata provenientes de poblaciones nativas de Año Nuevo $\left(h^{2}=0,78\right)$, siendo menor para familias provenientes de programas de mejoramiento genético de Australia $\left(h^{2}=0,34\right)$, Chile $\left(h^{2}=0,34\right)$ y Nueva Zelanda $\left(h^{2}=049\right)$ (Matheson et al. 2006). CAMCORE (Central America and Mexico Coniferous Resources Cooperative), ha evaluado la resistencia a $F$. circinatum en genotipos mejorados de $P$. radiata de Chile y Nueva Zelanda, determinándose mayor susceptibilidad entre las familias provenientes de Chile (Hodge \& Dvorak 2000, Matheson et al. 2006). Si bien el mejoramiento de especies forestales para resistencia a enfermedades es a largo plazo, constituye la estrategia costo-efectiva para reducir las pérdidas futuras en las plantaciones forestales. Debido a lo anterior, todos los estudios que permitan caracterizar la herencia genética de la resistencia a esta enfermedad son de gran importancia y permitirán continuar utilizando $P$. radiata en la producción mundial de maderas y fibra para la industria de la celulosa y papel (Gordon et al. 2001).

Situación actual de Fusarium circinatum en Chile

En Chile, las empresas forestales en conjunto con el Servicio Agrícola y Ganadero (SAG) han establecido medidas fitosanitarias obligatorias para los viveros con presencia de $F$. 
circinatum (denominados "viveros positivos"), indicadas en decretos y resoluciones del SAG, concordante al estatus de plaga cuarentenaria para $F$. circinatum (SAG 2013a). Estas incluyen muestreo de plantas previo a su traslado a terreno, desinfestación del suelo o substrato mediante incineración o tratamiento térmico, desinfestación de las herramientas de corte con hipoclorito de sodio o de calcio al $1 \%$ o peróxido de hidrógeno $\left(5 \mathrm{~g} \mathrm{~L}^{-1}\right)$ por $30 \mathrm{~s}$, y medidas de bioseguridad para vehículos, como la utilización de rodiluvios para la desinfección de neumáticos (Beéche et al. 2005, SAG 2011). Estas medidas de control están bajo control oficial para los viveros o campos de setos con detección del patógeno (SAG 2011). A partir del año 2003 los "viveros positivos" (Tabla I), son sometidos a control oficial, aplicando en forma obligatoria las medidas fitosanitarias establecidas para estos casos en la normativa vigente. Para la autorización de la movilización de plantas desde el vivero se debe certificar la ausencia de la enfermedad en el lote respectivo, realizando un muestreo en el vivero (dividido por lotes de plantas) por personal acreditado, colectando plantas con síntomas o asintomáticas. La detección e identificación del patógeno en laboratorio SAG o acreditado se realiza mediante taxonomía tradicional (Fig. 2) y técnicas de PCR. En caso de no cumplirse la normativa vigente, las plantas del lote positivo deben ser destruidas. Para la importación de semillas de especies hospederas al patógeno, las semillas deben llegar desinfectadas con fungicidas, se efectúan aislamientos en medios de cultivo selectivos y son mantenidas en cuarentena de post-entrada predial durante un periodo mínimo de un año (SAG 2005).

TABLA I. Número de viveros bajo control oficial de Fusarium circinatum (Fuente: SAG 2013b).

TABLE I. Nurseries under official control of F. circinatum (Source SAG 2013b).

\begin{tabular}{|c|c|c|c|c|c|c|c|c|c|c|c|}
\hline \multirow[t]{2}{*}{ REGIÓN } & \multirow{2}{*}{$\begin{array}{c}\mathrm{N}^{\circ} \text { DE VIVEROS CON } \\
\text { ESPECIES HOSPEDERAS } \\
(2012)\end{array}$} & \multicolumn{10}{|c|}{ NúMERO DE VIVEROS BAJO CONTROL OFICIAL ** } \\
\hline & & 2004 & 2005 & 2006 & 2007 & 2008 & 2009 & 2010 & 2011 & 2012 & 2013 \\
\hline O’Higgins* & 16 & 1 & 1 & 1 & 2 & 2 & 3 & 4 & 3 & 3 & 3 \\
\hline Maule* & 37 & 2 & 2 & 4 & 5 & 6 & 6 & 7 & 7 & 8 & 8 \\
\hline Biobío & 96 & 5 & 5 & 6 & 8 & 9 & 12 & 16 & 20 & 21 & 23 \\
\hline La Araucanía & 42 & 0 & 0 & 0 & 1 & 3 & 3 & 3 & 3 & 3 & 3 \\
\hline Los Ríos & 5 & 1 & 1 & 1 & 1 & 1 & 1 & 1 & 2 & 2 & 2 \\
\hline Total & 266 & 9 & 9 & 12 & 17 & 21 & 25 & 31 & 35 & 37 & 39 \\
\hline
\end{tabular}

* Los años 2011 y 2013 se levantaron las medidas de control obligatorio en viveros de la región por cumplir tres temporadas consecutivas sin detección del patógeno. / Mandatory control measurements in the region were discontinued in 2011 and 2013 after three consecutive seasons without pathogen detection.

** En la Región de la Araucanía, dos viveros bajo control oficial han cerrado. En la Región del Maule, un vivero de bajo C.O. ha cerrado. / In the Araucania Region, two nurseries, under official control have been closed. In Maule Region, one nursery under official control has closed.

TABLA II. Número (miles) y porcentaje de plantas eliminadas en relación del total de plantas movilizadas de especies hospederas a Fusarium circinatum en cinco regiones del país (Fuente: SAG 2013b).

TABLE II. Number (thousands) and percentage of plants eliminated in relation to the total mobilized plant host species of $F$. circinatum in five regions of Chile. (Source SAG 2013b).

\begin{tabular}{|c|c|c|c|c|c|}
\hline \multirow[t]{2}{*}{ AÑo } & \multicolumn{5}{|c|}{ REGIÓN } \\
\hline & $\begin{array}{c}\text { O'HIGGINS } \\
N^{\circ}(\%)\end{array}$ & $\begin{array}{l}\text { MAULE } \\
\mathrm{N}^{\mathrm{o}}(\%)\end{array}$ & $\begin{array}{l}\text { Biовío } \\
\mathrm{N}^{0}(\%)\end{array}$ & $\begin{array}{c}\text { LA ARAUCANÍA } \\
\mathrm{N}^{\mathrm{o}}(\%)\end{array}$ & $\begin{array}{c}\text { Los Ríos } \\
\mathrm{N}^{\mathrm{o}}(\%)\end{array}$ \\
\hline 2006 & 0 & $5(0,03)$ & $67(0,1)$ & 0 & 0 \\
\hline 2007 & 0 & $905(4,20)$ & $135(0,2)$ & 0 & 0 \\
\hline 2008 & 0 & $1.096(3,80)$ & $573(0,8)$ & 0 & 0 \\
\hline 2009 & 0 & $217(0,80)$ & $187(0,4)$ & 0 & $92(1,1)$ \\
\hline 2010 & $44(2,3)$ & $252(0,90)$ & $106(0,2)$ & $26(3,2)$ & $150(1,8)$ \\
\hline 2011 & 0 & 0 & $55(0,1)$ & 0 & $47(0,4)$ \\
\hline 2012 & $106(7,2)$ & 0 & $240(0,7)$ & 0 & $25(0,1)$ \\
\hline
\end{tabular}

$(+)$ : Plantas eliminadas corresponden a lotes positivos a F. circinatum. / Eliminated plants correspond to positive lots to F. circinatum. 


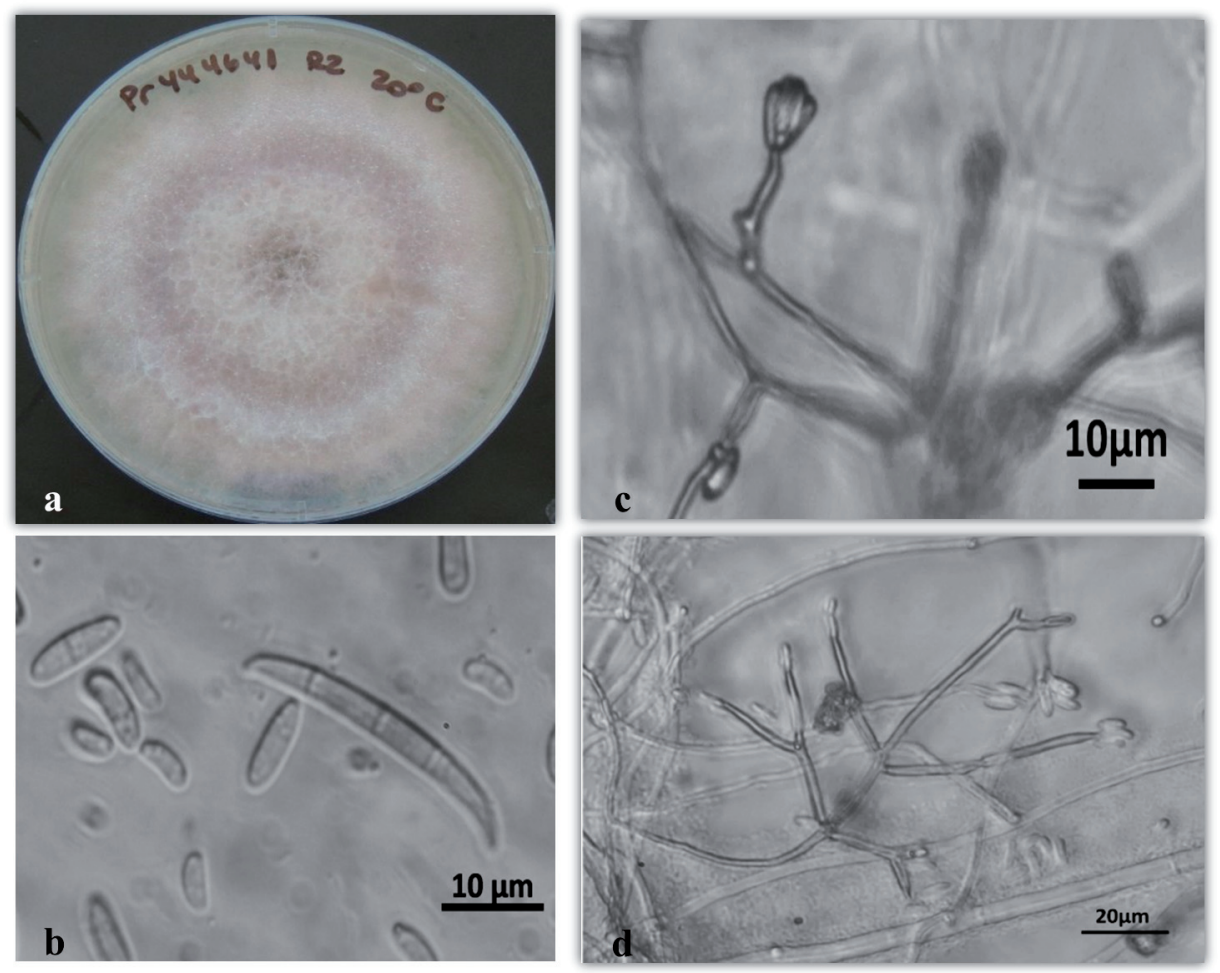

Figura 2. Características morfológicas de un aislado chileno de Fusarium circinatum. a: Colonia de F. circinatum creciendo en medio Agarpapa-dextrosa; b: microconidias y macroconidias, escala $=10 \mu \mathrm{m}$; : microconidias agrupadas en falsas cabezas, escala $=10 \mu \mathrm{m}$; $\mathrm{d}$ : grupo de fiálides, escala $=20 \mu \mathrm{m}$. Escala es indicada por barra (E. Sanfuentes).

FIGURE 2. Morphological characteristics of a Chilean strain of Fusarium circinatum. a: Colony of $F$. circinatum growing on potato-dextroseagar medium; b: microconidias and macroconidias, bar $=10 \mu \mathrm{m}$; c: microconidias accumulated in false heads, bar $=10 \mu \mathrm{m}$; d: phialides group, $b a r=20 \mu \mathrm{m}$. Scale is indicated by bar.

Todas las medidas de control tienen el objetivo de minimizar las pérdidas en vivero y disminuir el riesgo de traslado del patógeno, desde el vivero a las plantaciones. Algunos viveros positivos a $F$. circinatum, han permanecido por más de tres temporadas sin detecciones del patógeno, la mayoría presenta bajos niveles de infestación y otros han dejado de producir plantas (SAG 2013b). En los viveros positivos, sometido a control oficial, el número de plantas destruidas debido a la detección del patógeno ha disminuido en los últimos cuatro años, asociado principalmente a la aplicación de las medidas fitosanitarias implementadas (Tabla II).

En evaluaciones realizadas en cinco viveros positivos a $F$. circinatum el año 2004, no se detectó la presencia de conidias del patógeno en el aire y agua de riego. Se plantea que la principal forma de ingreso del patógeno a los viveros sería fundamentalmente vía substrato infestado (compost de corteza de pino), sustentándose esta posibilidad en la detección de $F$. circinatum en una empresa productora de substrato. Permanece como una incógnita cómo serían infestados estos lotes de compost, considerando que la corteza es obtenida desde plantaciones adultas cosechadas en donde el patógeno no está presente (Proyecto Fondo SAG 2007).
Actualmente, no existen tratamientos a base de fungicidas que sean completamente eficaces contra $F$. circinatum, aunque en ensayo in vitro los fungicidas difenoconazol, tebuconazol y fludioxinil inhiben el crecimiento micelial del patógeno (Proyecto Fondo SAG 2007). Hoy en día se utilizan fungicidas con los ingredientes activos captan (140-200 $\left.\mathrm{g} \mathrm{L}^{-1}\right)$, tebuconazol $\left(0,75-1,0 \mathrm{~L} \mathrm{ha}^{-1}\right)$ y azoxistrobina (200-400 $\left.\mathrm{cm}^{3} \mathrm{~L}^{-1}\right)$, donde solo este último está específicamente registrado contra $F$. circinatum (SAG 2012). Los otros fungicidas son para el manejo de hongos de amplio espectro dentro del cultivo de $P$. radiata.

Nuevas herramientas biotecnológicas están siendo desarrolladas por universidades, conjuntamente con empresas forestales, cuyo principal objetivo es apoyar la selección temprana de genotipos de $P$. radiata resistentes a F. circinatum. Entre los principales resultados obtenidos está la propuesta de un método de inoculación para seleccionar genotipos resistentes al patógeno en $P$. radiata y la evaluación de la expresión relativa de siete genes candidatos relacionados con la defensa en plantas (Donoso et al. 2015). Además, se han detectado regiones del ADN con efectos cuantitativos en la respuesta de las plantas frente al patógeno (Moraga-Suazo et al. 2014), entregando antecedentes para 
iniciar una búsqueda de genes candidatos relacionados con la resistencia de $P$. radiata a $F$. circinatum. Investigaciones similares son desarrolladas por otros grupos, que trabajan con especies forestales de importancia comercial, como por ejemplo TreeGenes (Wegrzyn et al. 2012), USDA Forest Service Research and Development (USFSR\&D 2013) y Forest Health Initiative (FHI 2009).

Amenaza potencial en CHILE

Si bien la enfermedad caracterizada como cancro resinoso aún no ha sido observada en plantaciones comerciales, el patógeno se encuentra en viveros y plantas de setos de $P$. radiata. Se presume que la mortalidad provocada por el patógeno en plantaciones de $P$. radiata menores a cuatro años estaría relacionada a plantas asintomáticas e infectadas con $F$. circinatum provenientes de viveros, similar a lo acontecido en plantaciones de $P$. patula en Sudáfrica. En ese país, la supervivencia de plántulas de $P$. patula se redujo de $88 \%$ en el año 2000 a un $64 \%$ en el 2007 (Mitchell et al. 2012), otras estimaciones indican una mortalidad de 25 $\%$ en el primer año de plantación (Crous 2005), planteando que la mortalidad en el campo es el resultado de plantas contaminadas o infectadas en viveros (Mitchell et al. 2011). En Chile, las pérdidas en viveros son variables y la enfermedad se encuentra bajo regulaciones establecidas por el SAG. En el año 2005 se reportaron pérdidas en huertos de plantas madres alcanzando en algunos viveros sobre el $40 \%$ y en otros se ha mantenido cerca del $20 \%$. En la producción de plantas, las pérdidas son menores, alcanzando hasta $14 \%$ de la producción (CPF 2005). Los lotes de plantas eliminadas por presencia de $F$. circinatum corresponden en muchos casos a genotipos elite con un alto valor para la industria forestal, generando una alta pérdida, asociado también a la necesidad de una mayor producción de estos genotipos. Debido a la eliminación de plantas, las pérdidas económicas causadas por este patógeno se estiman en alrededor de 540 mil dólares, correspondiente a la eliminación de 4,3 millones de plantas $(0,65 \%$ de la producción total $)$ entre los años 2006 y 2012. Si bien las pérdidas directas de plantas en los últimos años se han reducido (Tabla 2), el riesgo que la enfermedad tenga un desarrollo como cancro resinoso en plantaciones permanece latente.

En Chile se desconocen las razones por las cuales el cancro resinoso no ha ocurrido en plantaciones comerciales, especialmente aquellas establecidas en la inmediata vecindad de los viveros donde el patógeno está presente. Esta situación podría deberse en parte a la oportuna y rigurosa aplicación de las medidas de control que tiene como objetivo evitar la diseminación del patógeno. Por otro lado, también es posible que las condiciones climáticas del país no permitan el establecimiento y dispersión de la enfermedad en las plantaciones, debido a las bajas temperaturas y baja humedad ambiental en las zonas donde se ha reportado la presencia del patógeno (Wingfield et al. 2008). Estudios utilizando el software CLIMEX indicaron que en Chile no se presentan las condiciones óptimas para el desarrollo de la enfermedad (Ganley et al. 2009). Sin embargo, el cambio climático podría generar condiciones propicias para el establecimiento y desarrollo de la enfermedad en plantaciones comerciales.

Para el desarrollo del cancro resinoso deben existir componentes claves como son: heridas en la planta hospedera, condiciones de humedad y temperaturas adecuadas (Gordon 2001). Por otra parte, lesiones causadas por prácticas silvícolas también pueden facilitar la infección (Wingfield et al. 2008). En Chile están ausentes los insectos que causan heridas y permiten la diseminación del patógeno, tales como Pissodes nemorensis Germar y Rhyacionia frustrana Comstock presentes en el sureste de EE.UU. y los insectos nativos de California Conophthorus radiatae Hopkins, Ernobius punctulatus LeConte y Pityophyhorus spp. (Gordon et al. 2001), aunque los insectos Rhyacionia buoliana Den. \& Schiff e Hylugus ligniperda Fabricius, presentes en Chile, podrían transportar esporas del hongo y causar heridas en el árbol permitiendo la penetración del patógeno (Proyecto Fondo SAG 2007). Recientemente se detectó a Pissodes castaneus De Geer sobre trozas cebo de Pinus ponderosa Douglas ex Lawson en la comuna de Futaleufú, insecto que puede actuar como vector del patógeno (SAG 2013c). Por otra parte, lesiones causadas por prácticas silvícolas también pueden facilitar la infección (Wingfield et al. 2008).

En los países en que ha sido detectado el cancro resinoso en las plantaciones, existe coincidencia que la estrategia de control más adecuada es el uso de genotipos con mayores niveles de resistencia. Para $P$. radiata, así como para otras especies de Pinus, la selección genética efectuada en condiciones de invernadero es la técnica más rápida y sencilla disponible para los mejoradores, permitiendo discriminar con precisión las familias o clones resistentes de los susceptibles (Kayihan et al. 2005, Matheson et al. 2006, Roux et al. 2007). En Chile se han realizado inoculaciones controladas para determinar la resistencia al patógeno en diversas familias de $P$. radiata, detectándose variaciones fenotípicas en respuesta a la infección. Sin embargo, esta estrategia de selección basada en criterios sanitarios aún no es extendida a los programas de mejoramiento de las empresas forestales, siendo comúnmente priorizados características tales como crecimiento, forma, calidad de madera y rendimiento volumétrico.

Desde que $F$. circinatum fue identificado por primera vez en Chile, sólo se ha realizado un estudio con seis aislados del patógeno recolectados desde cuatro viveros, estableciéndose que estarían presentes los dos tipos de apareamientos (MAT-1 y MAT-2) (Jacobs et al. 2007). Un estudio efectuado en la misma época no tuvo éxito en determinar el número de GCVs presentes en el país (Proyecto Fondo SAG 2007). Recientemente, un trabajo 
utilizando marcadores microsatélites, empleando 10 aislados del patógeno provenientes de Chile, constató una baja diversidad genotípica del hongo $(D=0,3)$ y una nula posibilidad de que exista recombinación, indicando una reciente introducción del patógeno (Berbegal et al. 2013), semejante a la situación de California (Correll et al. 1992). No obstante, resultados de inoculaciones en plantas de $P$. radiata de diferentes edades, demostraron variación en agresividad entre aislados del patógeno, indicando una heterogeneidad para esta característica en la poblaciones de $F$. circinatum, siendo un factor de relevancia a considerarse en un programa de selección de germoplasma resistente a $F$. circinatum (Proyecto Fondo SAG 2007). Estos antecedentes aún son insuficientes para conocer con precisión la diversidad genética de la población del patógeno en Chile y su relación con otras poblaciones en otros países, siendo necesario aumentar significativamente el número de aislamientos para estudios futuros. El conocimiento de la genética poblacional de $F$. circinatum permitirá; por ejemplo, confirmar la estructura clonal de la población y el tipo de reproducción predominante; estos antecedentes son necesarios para cualquier programa de mejoramiento genético de resistencia al cancro resinoso del pino.

\section{CONCLUSIÓN}

A pesar de la distribución mundial del cancro resinoso del pino, existen muchas áreas donde se cultivan especies susceptibles y aún no han sido infectados por el patógeno. En consecuencia, las restricciones cuarentenarias siguen siendo un método importante de control. Si bien en Chile, la enfermedad producida por el hongo $F$. circinatum es un problema restringido sólo a viveros, existe un riesgo potencial de que el patógeno se propague a las plantaciones comerciales de $P$. radiata. Debido a la importancia de ésta especie en la economía del país, así como otras especies hospederas, tales como $P$. ponderosa y $P$. menziesii que son plantadas a nivel nacional, es imprescindible continuar aplicando las medidas fitosanitarias implementadas por el SAG y las empresas forestales, y especialmente desarrollar nuevas tecnologías para el control del patógeno. Todas las evidencias indican que el manejo integrado de la enfermedad, que actualmente está focalizada en viveros, es la estrategia más adecuada para reducir el riesgo que el cancro resinoso se extienda hacia las plantaciones comerciales de $P$. radiata, como también deberá ser para reducir el daño potencial una vez establecida la enfermedad, siendo necesario abordar medidas a corto y largo plazo, donde la resistencia genética debe ser la base del desarrollo futuro. Los problemas en el control de esta enfermedad cuarentenaria del pino y las pérdidas económicas asociadas al sector forestal ponen en evidencia la importancia de promover una comprensión sobre la biología y epidemiología del patógeno en las zonas del país donde se establece $P$. radiata, las que en gran parte aún permanecen sin respuesta.

\section{AGRADECIMIENTOS}

Se agradece el apoyo del Servicio Agrícola y Ganadero por facilitar información relevante para el estudio. A. Carrasco agradece a la Beca Doctoral CONICYT Nº 21090780.

\section{BIBLIOGRAFÍA}

Alonso, R. \& L. Bettucci. 2009. First report of the pitch canker fungus Fusarium circinatum affecting Pinus taeda seedlings in Uruguay. Australasian Plant Disease 4: 91-92.

Barrows-Broaddus, J. \& T.J. KerL. 1981. Inhibition of Fusarium moniliforme var. subglutinans, the causal agent of pine pitch canker, by the soil bacterium Arthrobacter sp. Canadian Journal of Microbiology 27: 20-27.

Beéche, M., P. González, S. Sandoval \& M.E. Murillo. 2005. Informativo fitosanitario forestal. Servicio Agrícola y Ganadero 1: 1-5.

Berbegal, M., A. Pérez-Sierra, J. Armengol \& N.J. Grünwald. 2013. Evidence for multiple introductions and clonality in Spanish populations of Fusarium circinatum. Phytophatology 103: 851-861.

Bragança, H., E. Diogo, F. Moniz \& P. Amaro. 2009. First report of pitch canker on pines caused by Fusarium circinatum in Portugal. Plant Disease 93: 1079.

Britz, H., T.A. Coutinho, B.D. Wingfield, W.F.O. Marasas \& M.J. WingField. 2005. Diversity and differentiation in two populations of Gibberella circinatum in South Africa. Plant Pathology 54: 46-52.

Carlucci, A., L. Colatruglio \& S. Frisullo. 2007. First report of pitch canker caused by Fusarium circinatum on Pinus halepensis and P. pinea in Apulia (southern Italy). Plant Disease 91(12): 1683.

Cook, D.C. \& A.C. MAtheson. 2008. An estimate of the potential economic impact of pine pitch canker in Australia. Australian Forestry 71: 107-112.

Correll, J.C., T.R. Gordon \& A.H. McCain. 1992. Genetic diversity in California and Florida populations of the pitch canker fungus Fusarium subglutinans f. sp. pini. Phytopathology 82: 415-420.

Coutinho, T., E. Steenkamp, K. Mongwaketzi, M. Wilmot \& M. WingFIELD. 2007. First outbreak of pitch canker in South African pine plantation. Australasian Plant Pathology 36: 256-261

Crous, J.W. 2005. Post establishment survival of Pinus patula in Mpumalanga, one year after planting. Southern African Forestry Journal 205: 3-8.

Donoso, A., V. Rodriguez, A. Carrasco, R. Ahumada, E. Sanfuentes \& S. Valenzuela. 2015. Relative expression of seven candidate genes for pathogen resistance on Pinus radiata infected with Fusarium circinatum. Physiological and Molecular Plant Pathology 92: 42-50.

Dwinell, L.D., J. Barrows-Broaddus \& E.G. Kuhlman. 1985. Pitch canker: a disease complex of southern pines. Plant 
Disease 69: 270-276.

Dwinell, L.D., S.W. Fraedrich \& D. Adams. 2001. Diseases of pines caused by the pitch canker fungus. In: B.A. Summerell, J.F. Leslie, D. Backhouse \& W.L. Bryden \& L.W. Burgess (eds). Fusarium: Pp. 225-232. Paul E. Nelson Memorial Symposium' (The American Phytopathological Society: St Paul, MN).

EPPO (European and Mediterranean Plant Protection Organization). 2006. EPPO Reporting Service 2006-05 no. 104. URL: https://archives.eppo.int/EPPOReporting/2006/ Rse-0605.pdf Visto: Marzo 13, 2015.

FHI (Forest Health Initiative). 2009. URL: http:// foresthealthinitiative.org/Visto: Septiembre 10, 2013.

Fitza, K.N.E., K.G. Payn, E.T. Steenkamp, A.A. Myburg \& S. NAIDOO. 2013. Chitosan application improves resistance to Fusarium circinatum in Pinus patula. South African Journal of Botany 85: 70-78.

Fraedrich, B.R. \& W. Witcher. 1982. Influence of fertilization on pitch canker development on three southern pine species. Plant Disease 66: 938-940.

FSC (Forest Stewardship Council). 2007. FSC-GUI-30-001 Pesticide Policy: Guidance on Implementation. URL: https://ic.fsc.org/download.fsc-pesticides-policyguidance.a-625.pdf. Visto: Abril 15, 2013.

Ganley, R.J., M.S. Watt, L. Manning \& E. IturritXa. 2009. A global climatic risk assessment of pitch canker disease. Canadian Journal of Forest Research 39: 2246-2256.

Gordon, T.R., A.J. Storer \& D.L. Wood. 2001. The pitch canker epidemic in California. Plant Disease 85: 1128-1139.

Gordon T.R., S.C. Kirkpatrick, J.C. Petersen \& C.J. Friel. 2006. Potential diversity in vegetative compatibility groupings in the California population of Gibberella circinata. Mycological Research 110: 936-940.

Gordon, T.R., C.L. Swett \& M.J. Wingfield. 2015. Management of Fusarium diseases affecting conifers. Crop Protection 73: 28-39.

Guerra-Santos, J.J. 1999. Pitch canker on Monterey pine in Mexico. In: M.E. Devey, A.C. Matheson, T.R. Gordon (eds.) Current and potential impacts of pitch canker in radiata pine. pp. 58-61. Proceedings of the Impact Monterey Workshop. California, USA, 30 noviembre - 3 diciembre 1998, Kingston ACT, CSIRO Australia.

Hepting, G.H. \& E.R. Roth. 1946. Pitch canker, a new disease of southern pines. Journal of Forestry 44: 742-744.

Hepting, G.H. \& E.R. Roth. 1953. Host relations \& spread of the pine pitch canker disease. Phytopathology 43: 475.

Hodge, G.R. \& W.S. Dvorak. 2000. Differential responses of Central American and Mexican pine species and Pinus radiata to infection by the pitch canker fungus. New Forests 19: 241-258.

Huss, M.J., C.L. Campbell, B.D. Jennings \& J.F. Leslie. 1996. Isozyme variation among biological species in the Gibberella fujikuroi species complex (Fusarium section Liseola). Applied and Environmental Microbiology 63: 3750-3756.

Iturritxa, E., R.J. Ganley, J. Wright, E. Heppe, E.T. Steenkamp, T.R. Gordon, M.J. Wingfield. 2011. A genetically homogenous population of Fusarium circinatum causes pitch canker of Pinus radiata in the Basque Country, Spain. Fungal Biology 115: 288-295.
Jacobs, A., T.A. Coutinho, M.J. Wingfield, R. Ahumada \& B.D. WINGFIELD. 2007. Characterization of the pitch canker fungus, Fusarium circinatum, from Chile. South African Journal of Science 103: 253-257.

Kayihan, G.C., D.A. Huber, A.M. Morse, T.L. White \& J.M. DAVIS. 2005. Genetic dissection of fusiform rust \& pitch canker disease traits in loblolly pine. Theoretical \& Applied Genetics 110: 948-958.

Kobayashi, T. \& M. Muramoto. 1989. Pitch canker of Pinus luchuensis, a new disease of Japanese forests. Forest Pests 40: 169-173.

Kuhlman, E.G. 1982. Varieties of Gibberella fujikuroi with anamorphs in Fusarium section Liseola. Mycologia 74: 759-768.

Landeras, E, A.P. Garcia, Y. Fernandez \& M. Braña. 2005. Outbreak of Pitch Canker caused by Fusarium circinatum on Pinus spp. in Northern Spain. Plant Disease 89: 1015.

Martínez-Álvarez, P., R.A. Fernández-GonzÁlez, A. V. SanzRos, V. Pando \& J.J. Diez. 2016. Two fungal endophytes reduce the severity of pitch canker disease in Pinus radiata seedlings. Biological Control 94: 1-10.

Matheson, A.C., M.E. Devey, T.R. Gordon, W. Werner, D.R. Vogler, C. BALocchi \& M.J. CARson. 2006. Heritability of response to inoculation by pine pitch canker of seedlings of radiata pine. Australian Forestry Journal 70: 101-106.

Mitchell, R.G., E.T. Steenkamp, T.A. Coutinho \& M.J. Wingfield. 2011. The pitch canker fungus, Fusarium circinatum: implications for South African forestry, Southern Forests: Journal of Forest Science 73: 1-13.

Mitchell, R.G., T.A. Coutinho, E. Steenkamp, M. Herbert \& M.J. Wingfield. 2012. Future outlook for Pinus patula in South Africa in the presence of the pitch canker fungus (Fusarium circinatum). Southern Forests: A Journal of Forest Science 74: 203-210.

Moraga-Suazo, P., A. Opazo, S. Zaldúa, G. González \& E. SANFuentes. 2011. Evaluation of Trichoderma spp and Clonostachys spp strains to control Fusarium circinatum in Pinus radiata seedlings. Chilean Journal of Agricultural Research. 71: 412-417.

Moraga-Suazo, P., L. Orellana, P. Quiroga, C. Balocchi, E. Sanfuentes, R.W. Whetten, R. Hasbún \& S. Valenzuela. 2014. Development of a genetic linkage map for Pinus radiata and detection of pitch canker disease resistance associated QTLs. Trees 28: 1823-1835.

Morse, A.M., C.D. Nelson, S.F. Covert, A.G. Holliday, K.E. SMith \& J.M. Davis. 2004. Pine genes regulated by necrotrophic fungus Fusarium circinatum. Theoretical \& Applied Genetics 109: 922-932.

Nelson, P.E., T.A. Toussoun \& W.F.O. Marasas. 1983. 'Fusarium species: an illustrated manual for identification.' (Pennsylvania State University Press: University Park, PA). 193 pp.

Nirenberg, H.I. \& K. O’Donnell. 1998. New Fusarium species and combinations within the Gibberella fujikuroi species complex. Mycologia 90: 434-458.

Perry, J.P., A. Graham \& D.M. Richardson. 1998. Pine ecology and biogeography-an introduction. In: D.M. Richardson (eds), Ecology and Biogeography of Pinus, pp. 137-149. Cambridge University Press, Cambridge, UK.

Pfenning, L.H., S.D.S. Costa, M.P.D. Melo, H. Costa, J.A. 
Ventura, C.G. Auer \& Á.F.D. Santos. 2014. First report and characterization of Fusarium circinatum, the causal agent of pitch canker in Brazil. Tropical Plant Pathology 39: 210-216.

Proyecto INNOVA Bio-Bio (Noviembre 2009 - Mayo 2013). Desarrollo de herramientas biotecnológicas para el control de Fusarium circinatum en viveros de Pinus radiata. URL: http://www2.udec.cl/patfor/images/stories/documentos/ Proyecto_Innovabiobio_08-PCS1-312.pdf. Visto: Abril 15,2015

Proyecto Fondo SAG, Informe Final (Noviembre 2003-Abril 2007) (Código C3-72-08-12). 2007. Fusarium circinatum: Conocimiento de patógeno y establecimiento de bases para su control en Pinus radiata. URL: http://www.sag.cl/sites/ default/files/INFORME\%20\%20FINAL\%20CPF\%20 Fusarium\%20C3-72-08-12.pdf

Quesada, T., V. Gopal, W.P. Cumbie, A.J. Eckert, J.L. Wegrzyn, D.B. Neale, B. Goldfarb, D.A. Huber, G. Casella \& J.M. DAvis. 2010. Association Mapping of Quantitative Disease Resistance in a Natural Population of Loblolly Pine (Pinus taeda L.). Genetics 186: 677-686.

Roux, J., B. Eisenberg, A. Kanzler, A. Neil, V. Coetzee, E. Keitzka \& M.J. WingfielD. 2007. Testing of selected South African Pinus hybrids \& families for tolerance to the pitch canker pathogen, Fusarium circinatum. New Forest 33: 109-123.

Runion, G.B., S.C. CADE \& R.I. BRUCK. 1993. Effects of carbofuran and thiabendazole on incidence of pitch canker of loblolly pine. Plant Disease 77: 166-169.

SAG (Servicio Agrícola y Ganadero). 2005. Resolución N ${ }^{\circ}$ 707: Establece requisitos fitosanitarios para el ingreso de semillas de especies forestales. Santiago, Chile.

SAG (SErvicio Agrícola y Ganadero). 2011. Resolución No 1754: Modifica Resolución $\mathrm{N}^{\circ} 1.424$ de 2008 que establece medidas de control obligatorio de F. circinatum. Santiago, Chile.

SAG (Servicio Agrícola y Ganadero). 2012. URL: http://www. sag.cl/content/lista-de-plaguicidas-autorizados Visto: Junio 09, 2012.

SAG (Servicio Agrícola y Ganadero). 2013a. Resolución N ${ }^{o}$ 1162: Modifica Resolución No 3.080 de 2003 que establece criterios de regionalización, en relación a las plagas cuarentenarias para el territorio de Chile. Santiago, Chile.

SAG (Servicio Agrícola y Ganadero). 2013b. Carta No 1729. URL: http://custodiafirma1305.acepta.com/v01/ f4e28438bbca18bacc9b57a1 eae4f6386b674ca9

SAG (Servicio Agrícola y Ganadero). 2013c. Primera detección en chile del "gorgojo de la corteza del pino" Pissodes castaneus (col: Curculionidae). Santiago, Chile. URL: http://www.sag.cl/sites/default/files/informe-pissodes.pdf Visto: Junio 09, 2013.

Schweigkofler, W., K. O’Donnell \& M. Garbelotto. 2004. Detection and quantification of Fusarium circinatum, the causal agent of pine pitch canker, from two California sites by using a real-time PCR approach combined with a simple spore trapping method. Applied and Environmental Microbiology 70: 3512-3520.
SLINSKI, S.L., S.C. KIRKPATRICK \& T.R. Gordon. 2016. Inheritance of virulence in Fusarium circinatum, the cause of pitch canker in pines. Plant Pathology. doi:10.1111/ppa.12511

Snyder, W.C., E.R. Toole \& G.H. Hepting. 1949. Fusaria associated with mimosa wilt, sumac wilt and pine pitch canker. Journal of Agricultural Research 78: 365-382.

Steenkamp, E.T, C.A. Roads, M. Kvas \& M.J. Wingfield. 2012. Fusarium circinatum and pitch canker of Pinus in Colombia. Australian Plant Pathology 41: 483-491.

Storer, A.J., T.R. Gordon \& S.L. Clark. 1998. Association of the pitch canker fungus, Fusarium subglutinans f. sp. pini with Monterey pine seeds, and seedlings in California. Plant Pathology 47: 649-656.

Storer, A.J., D.L. Wood \& T.R. Gordon. 2002. The epidemiology of pitch canker of Monterrey pine in California. Forest Science 48: 694-700.

Thoungchaleun, V., K.W. Kim, D.K. Lee, C.S. Kim \& E.H. Park. 2008. Pre-Infection Behavior of the Pitch Canker Fungus Fusarium circinatum on Pine Stems. Plant Pathology Journal 24: 112-117.

TPCP. 2002. Fusarium circinatum in pine nurseries: A guide to appropriate management strategies. Tree Pathology Cooperative Programme (TPCP), University of Pretoria, Pretoria, South Africa. URL: http://www.fabinet.up.ac.za/ src.fabinet.up.ac.za/tpcp/pamphlets/fusarium.pdf Visto: Abril 15, 2015.

USFSR\&D. 2013. US Forest Service Research \& Development. URL: http://www.fs.fed.us/research/index.php Visto: Septiembre 10, 2013.

Viljoen, A., M.J. Wingfield \& W.F.O. Marasas. 1994. First report of Fusarium subglutinans f. sp. pini on seedlings in South Africa. Plant Disease 78: 309-312.

Wegrzyn, J.L., D. Main, B. Figueroa, M. Choi, J. Yu, D.B. Neale, S. Jung, T. Lee, M. Stanton, P. Zheng, S. Ficklin, I. Cho, C. Peace, K. Evans, G. Volk, N. Oraguzie, C. Chen, M. Olmstead, G. Gmitter \& A.G. Аввотt. 2012. Uniform standards for genome databases in forest and fruit trees. Tree Genetics \& Genomes 8: 549-557.

WikLer, K. \& T.R. Gordon. 2000. An initial assessment of genetic relationships among populations of Fusarium circinatum in different parts of the world. Canadian Journal of Botany 78: 709-717.

Wingfield, M.J., A. Jacobs, T.A. Coutinho, R. Ahumada \& B.D. WingFIELD. 2002. First report of the pitch canker fungus, Fusarium circinatum, on pines in Chile. Plant Pathology 51: 397.

Wingfield, M.J., A. Hammerbacher, R.J. Ganley, E.T. Steenkamp, T.R. Gordon, B.D. Wingfield \& T.A. Coutinho. 2008. Pitch canker caused by Fusarium circinatum - a growing threat to pine plantations and forests worldwide. Australasian Plant Pathology 37: 319-334.

Woo, K.S., J.H. Yoon, S.U. Han \& C.S. KIM. 2010. Assessment of pathogenic variation against pitch canker pathogen, Fusarium circinatum in Pinus thunbergii and responses of natural selection Pinus x Rigitaeda to branch inoculation in a seed orchard. Plant Pathology Journal 26: 299-305. 